\title{
L'expression du doute dans les mystères de la Passion de la fin du Moyen Âge ou la mise en actes et en paroles du libre-arbitre
}

Vanessa Mariet-Lesnard

\section{(2) OpenEdition}

\section{Journals}

Édition électronique

URL : http://journals.openedition.org/questes/1234

DOI : 10.4000/questes. 1234

ISSN : 2109-9472

Éditeur

Les Amis de Questes

\section{Édition imprimée}

Date de publication : 15 mars 2012

Pagination : 104-120

ISSN : 2102-7188

\section{Référence électronique}

Vanessa Mariet-Lesnard, "L'expression du doute dans les mystères de la Passion de la fin du Moyen Âge ou la mise en actes et en paroles du libre-arbitre », Questes [En ligne], 23 | 2012, mis en ligne le 01 janvier 2014, consulté le 20 avril 2019. URL : http://journals.openedition.org/questes/1234 ; DOI : 10.4000/questes. 1234 


\title{
L'expression du doute dans les mystères de la Passion de la fin du Moyen Âge ou la mise en actes et en paroles du libre-arbitre
}

\author{
Vanessa MARIET-LESNARD
}

Le Moyen Âge est l'âge de la foi qui, si elle existe à travers un système de croyances et de pratiques complexe, exclut d'emblée la voie du doute absolu et ramène toute hésitation vers la certitude initiale : l'existence de Dieu. Ainsi, lorsque la représentation du mystère de la Passion montre quelques épisodes de l'Ancien Testament et la vie de Jésus pour «corroborer la foi du peuple néophyte ${ }^{1}$, elle laisse peu de place au doute. Si l'on se réfère au Dictionnaire de la $B_{i b l e}^{2}$, le terme «doute » partage la même étymologie que le terme «deux ». Il souligne alors une dualité dans la pensée, divisée entre deux directions sans que l'on puisse se décider pour l'une ou pour l'autre. En cela, le doute apparaît comme l'antithèse de la foi puisqu'il inclut la défiance de l'homme envers son Créateur. Cette conception interdit de prime abord de mettre en scène le doute dans un théâtre à sujet religieux, dont la visée est l'édification.

Pour autant, les grandes Passions de la fin du Moyen Âge ne se privent pas de représenter les épisodes bibliques traditionnels où les personnages doutent ou, pour le moins, hésitent. Plus encore, les auteurs, véritables hommes de théâtre, sont attentifs aux aspects intrinsèques de la représentation théâtrale qui reste fidèle à la lettre théologique, même s'ils créent bien autre chose pour le spectateur chrétien que des scènes

\footnotetext{
${ }^{1}$ Saint Ethelwold, Regularis Concordia, in E. K. Chambers, The Mediaeval Stage, Oxford, Clarendon Press, 1925 ( 1 ère éd. 1903), 2 tomes, t. II, appendice O, p. 306-309.

2 Dictionnaire Encyclopédique de la Bible. Les Choses, les Hommes, les Faits, les Doctrines, Alexandre WestPHAL (dir.), Paris, Éditions «Je sers », 1932, entrée « doute ».
} 
iconiques. Cette nouvelle considération du doute ou de l'hésitation sur le hourdement ${ }^{3}$ ne vient jamais contredire la définition première du terme ; au contraire, elle l'enrichit de nouveautés.

Ainsi, l'expression du doute dans les mystères de la Passion s'inscrit dans le paradigme christique comme le suggèrent les Écritures, mais sera finalement redéfinie, à l'inverse, dans le paradigme diabolique ${ }^{4}$. Reste l'épisode des doutes de Judas, épisode marginal qui fonctionne comme une charnière, tant au plan dramatique que théologique, ouvrant une perspective nouvelle au monologue de l'hésitation.

\section{Les doutes du paradigme christique}

Lorsque l'on évoque l'expression du doute dans les mystères de la Passion, l'épisode de Joseph est immédiatement convoqué parce qu'il est le symbole du questionnement du paradigme christique. Toutefois, il faut se souvenir du doute initial qui fonde de nombreuses caractéristiques de l'ensemble, car il est un modèle dramatique et théologique : le doute d'Ève. L'intervention monologuée de cette dernière chez Arnoul Gréban est brève mais propose des clés de compréhension pour les interventions similaires à venir dans l'œuvre :

\footnotetext{
${ }^{3}$ Les termes «hourdement » et « hourd» désignent l'ensemble du dispositif scénique qui permet la représentation théâtrale d'un mystère de la Passion.

${ }^{4}$ Le terme «paradigme » est défini par Anne Ubersfeld comme « un ensemble de signes théâtraux plus ou moins substituables les uns aux autres ou qui s'évoquent les uns les autres par métonymie. Les personnages sont des signes théâtraux pour l'analyse desquels le recours à cette notion s'avère particulièrement fécond » (Anne UBERSFELD, Lire le théâtre, Paris, Belin, «Belin sup. Lettres », 1996, 3 tomes, t. I, p. 132). Nous entendons le paradigme diabolique comme l'ensemble des référents, pris ensemble ou individuellement, qui renvoient aux diables, au Mal ou à l'enfer. À l'inverse, le paradigme christique s'attache aux référents opposés, ces deux sphères constituant le même paradigme chrétien dans lequel elles prennent place. En effet, la sphère diabolique n'acquiert jamais une indépendance totale vis-à-vis de la sphère divine, cette dernière tolérant la première au cœur d'un système plus global qui est le paradigme chrétien. Il est notable que la perméabilité que nous constaterons entre la représentation théâtrale et le public, constitué massivement, voire exclusivement, de spectateurs chrétiens, oblige parfois à intégrer le spectateur à ce paradigme chrétien.
} 
Voicy de beaulx arbres monjoye

Et de fruiz assez a choisir.

Je prens a les veoir grant plaisir,

Mais celluy, par especïal,

De scïence de bien et de mal

Me plaist moult. Et se j'en menjoye

Las, que dis je? je n'oseroye :

Dieu le nous deffend par ses dictz. ${ }^{5}$

Ève se parle à elle-même, elle s'interroge, mais son doute, sitôt énoncé, est rejeté et le personnage se plie à la discipline divine. L'écriture d'Arnoul Gréban est nouvelle à deux égards dans cet épisode : d'abord, c'est bien sûr une amplification de la Genèse ${ }^{6}$, mais, surtout, Ève génère elle-même le doute (car ce n'est qu'à la didascalie suivante que Satan apparaît sous la forme d'un serpent). Ainsi, l'intérêt de l'épisode chez Arnoul Gréban est dramatique puisqu'il suppose que le monologue se tient hors de la présence d'autres personnages ${ }^{7}$. C'est une situation nouvelle qui apparaît ici pour la première fois et qui reste un signal dramatique fort tout au long de la représentation pour le spectateur. En effet, plus tard, Joseph s'isole pour douter, Marie-Madeleine s'interroge seule et, plus subtilement, Marie hésite en marge des autres personnages. Ce monologue présente aussi un intérêt théologique nouveau : ce n'est pas le diable qui insuffle son doute et son envie à Ève, ils lui sont intrinsèques. Cette position souligne la volonté de l'auteur qui veut insister sur le fait que le chrétien qui doute est bien souvent isolé avec ce sentiment, que seule la foi peut éteindre. Arnoul Gréban, en particulier, montre le libre-arbitre humain en actes et en paroles. En effet, si finalement Ève pèche et fait le mauvais choix, le doute naît ici uniquement de son questionnement, là où d'autres œuvres font

${ }^{5}$ Le Mystère de la Passion d'Arnoul Gréban, Omer Jodogne (éd.), Bruxelles, Palais des Académies, 2 vol., 1965-1983, v. 463-470. Nous soulignons.

${ }^{6}$ Genèse, III, 1-7.

${ }^{7}$ Dans d'autres œuvres, notamment le Jeu d'Adam, l'hésitation d'Ève est le produit de la parole de Satan qui est omniprésent à ses côtés. Cf. Le Mystère d'Adam (Ordo representacionis Ade), Paul AEBISCHER (éd.), Genève/Paris, Droz/Minard, «Textes littéraires français », 99, 1964. 
immédiatement intervenir Satan. Arnoul Gréban insiste sur la culpabilité d'Ève. La première tentation n'est donc pas diabolique, elle est humaine, et Ève doute de sa propre parole : «Las, que dis je ? ${ }^{8}$.

La démarche de l'auteur du Jeu d'Adam est très différente. Ève dit en effet au personnage de Diabolus «Bien te pois creire a ta parole». Mais, plus loin, celui-ci insiste: «Manjue le; n'aiez dutance :/ Le demorer serrat emfance ${ }^{9}$. Il ne s'agit pas d'un monologue. Il reste que la décision d'Ève vient sans aucun doute d'elle-même puisque, juste avant, Adam, dans la même situation, a fait sèchement valoir son refus à Diabolus. Pire encore, Ève ne doute de rien du tout. Par là, elle annonce le doute infernal. Tout l'échange est encadré par des répliques diaboliques qui empêchent Ève d'hésiter face à la parole de Diabolus : c'est là que se situe la première faute d'Ève qui croit à cette parole infernale. La volonté permanente de Diabolus qui vise à évacuer toute possibilité de mise en cause de sa parole est le prédicat du discours et des actes diaboliques. Diabolus crée une nouvelle certitude pour sortir victorieux de cet échange : faire adopter au «tenté » un système qui n'est pas le sien mais qui le devient dans la certitude est le principe même de la tentation diabolique. Finalement, la certitude d'Ève est telle qu'elle détermine son attitude envers Adam lorsqu'elle lui dit: «Manjue! N'en poez doter $»^{10}$. En rejetant le doute, Ève adhère au discours de Diabolus et son geste revêt les atours du geste diabolique et la même dynamique que celui-ci - celle du «faire faire »comme le montre le parallèle entre le vers 275 («Manjue le, n’aiez dutance ») et le vers 314 (« Manjue ! N'en poez doter ») .

\footnotetext{
${ }^{8}$ Le Mystère de la Passion d'Arnoul Gréban, éd. cit., v. 469.

${ }^{9}$ Le Mystère d'Adam, éd. cit., v. 219 et 275-276. Nous soulignons.

${ }^{10}$ Ibid., v. 314 (nous soulignons). Nous aimerions rappeler que le verbe « douter » dans cette occurrence conserve toute l'ambivalence sémantique qui le caractérise au Moyen Âge : ainsi, il signifie à la fois « hésiter » et « craindre » ou « redouter ».
} 
Ces épisodes laissent donc s'exprimer le doute et sont autant de mises en actes et en paroles du libre-arbitre humain. Or, ce libre-arbitre permanent est également exercé par l'homme chrétien qui constitue le public. Les auteurs montrent ces personnages comme des exemples à suivre ou à ne pas suivre, en tout cas à méditer.

Pourtant, cette expression du doute, même si elle existe dans une visée d'édification du public, est à chaque fois une défiance envers Dieu. D'ailleurs, la limite à l'expression du doute est contenue toute entière dans les Écritures : qu'il s’agisse de Joseph, d'Ève ou de Judas, l'incapacité à choisir franchement la voie de Dieu ou celle du Mal est bornée par l'intervention de puissances supérieures. Par exemple, Gabriel ne tarde pas à guider la décision de Joseph, tout comme Satan dirige la main et 1'esprit d'Ève ou lorsque Lucifer ordonne que les diables harcèlent Judas. La limite que constitue l'apparition de ces instances supérieures met fin à l'atermoiement de la prise de décision, mais elle brise aussi un rythme scénique et réamorce l'action dramatique. Dans ces cas précis (sauf peutêtre pour Judas), les auteurs amplifient les Écritures mais respectent la Lettre, car si le libre-arbitre est un fait, il ne peut pas exister hors du cadre de la foi ${ }^{11}$. Puisque les fatistes (c'est-à-dire les auteurs de mystères) ne prennent pas tant de liberté du côté de l'expression théologique, regardons

\footnotetext{
${ }^{11}$ Saint Augustin le premier a rappelé que «la volonté libre sans laquelle personne ne peut bien vivre, tu dois reconnaître et qu'elle est un bien, et qu'elle est un don de Dieu, et qu'il faut condamner ceux qui mésusent de ce bien plutôt que de dire de celui qui l'a donné qu'il n'aurait pas dû le donner » (SAINT AUGUSTIN, De Libero arbitrio, in CEuvres de saint Augustin, vol. 6, Dialogues philosophiques, III, Goulven MADEC (éd. et trad.), Paris, Desclée de Brouwer, 1976, «Bibliothèque augustinienne », 6, II, 18, 48). Par ailleurs, saint Bernard a précisé dans La Grâce et le libre arbitre quelques différences entre le libre-arbitre qui appartient à tout homme et la liberté qui s'acquiert. Ainsi, selon saint Bernard, il existe trois sortes de liberté libératrice dont la première, libertas a necessitate, correspond au libre-arbitre « du fait que nécessaire s'oppose évidemment à volontaire : car ce qui se fait par nécessité ne relève plus de la volonté et inversement » (SAINT Bernard DE ClairvauX, La Grâce et le libre arbitre, Françoise CALlerot (intro. et trad.), Paris, Éditions du Cerf, «Sources Chrétiennes », 393, 1993, § 6, p. 258).
} 
du côté de la représentation théâtrale. Ainsi, lorsque Gabriel ou Satan apparaissent sur le hourdement, aux côtés du personnage qui doute, c'est l'incarnation d'un être spirituel sur la scène à l'instant du choix qui fait basculer tout à la fois l'action et la mise en scène par le rapprochement matérialisé entre la sphère divine et la sphère terrestre. En effet, au plan de la mise en scène, cela n'est pas sans nous rappeler les Ars moriendi où diables et anges surgissent aux pieds du mourant pour tirailler et se départager l'âme du chrétien trépassant. Donc, si les épisodes de doute dans le théâtre à sujet religieux sont des passages très fidèles aux Écritures, ils enrichissent le drame et sa représentation, offrent une épaisseur aux personnages et un écho aux représentations iconographiques familières aux spectateurs.

L'épisode des doutes d'Ève fonde et annonce des comportements typiques d'autres personnages du paradigme christique, notamment celui de Marie. Dans le mystère d'Arnoul Gréban, le planctus Mariae, qui est un morceau poétique parfaitement codifié $^{12}$, apparaît d'abord comme le discours de la plainte. Mais la voix double qui prend en charge ce discours permet de déceler l'expression du doute. Ainsi, ce sont tour à tour l'Ancilla de Dieu et la Mater Dolorosa qui prennent la parole. Or, si l'Ancilla de Dieu n'exprime que la certitude de la nécessité de la Passion, la Mère semble douter du dessein divin. Lorsqu'elle parle, elle évoque les hésitations qui l'assaillent à l'idée de la disparition prochaine de son fils ${ }^{13}$. La Mère qu'elle est remet également en question les promesses liées à la Passion qui ont jadis animé son attitude d'Ancilla : «Ou est celle joye, notree, / Que me promis en ton salu ? ${ }^{14}$. Outre le reproche qui point dans

12 Claude Thiry définit le planctus comme une forme souple qui respecte toutefois quelques codes rhétoriques: «elle doit faire appel aux procédés de pathos amplification, répétition, apostrophe, périphrase - et aux topoï du panégyrique » (Claude THIRY, La Plainte funèbre, Turnhout, Brepols, 1978, p. 36).

${ }^{13}$ Le Mystère de la Passion d'Arnoul Gréban, éd. cit., v. 27016-27033.

${ }^{14}$ Ibid., v. 27026-27027. 
cette réplique mariale, de véritables questions et doutes naissent. Pourtant, ils ne sont pas condamnables puisqu'ils sont le fondement d'une perspective théologique très importante : ces doutes n'empêchent jamais l'acceptation de la Passion. Toutefois, en filigrane du planctus Mariae transparaît la question universelle : pourquoi la Passion ? La Passion du Palatinus s'évertue (par la consolation du Fils ou le discours de Jean ${ }^{15}$ ) à rappeler qu'il s'agit de faire valoir l'intérêt universel contre l'intérêt individuel. Chez Arnoul Gréban, tant que Marie hésite, elle n'accepte jamais d'incarner la Mater Dolorosa. Cette hésitation, très bien décrite par Véronique Dominguez ${ }^{16}$, répond parfaitement à la notion « d'oscillation pendulaire » détaillée par Erich Auerbach ${ }^{17}$. C'est pourquoi nous insistons plus particulièrement sur le parallèle entre cette hésitation mariale et celle du Père lors du Procès de Paradis dans l'œuvre d'Arnoul Gréban ${ }^{18}$. Si les deux personnages émettent des doutes, chacun les tarit dans une acceptation souffrante : la Compassion de la Mère et la Passion du Père encadrent l'existence et la mort du Fils.

${ }^{15}$ La Passion du Palatinus, mystère du XIV siècle, Grace FrANCK (éd.), Paris, Champion, «Classiques Français du Moyen Âge», 30, 1972, v. 990-993 et v. 1116-1191.

${ }^{16}$ Véronique DomingueZ, La Scène et la Croix. Le jeu de l'acteur dans les Passions dramatiques françaises (XIV $-\mathrm{XVI}^{\mathrm{e}}$ siècles), Turnhout, Brepols, «Textes, Codex \& Contexte », 2, 2007, p. 252-284.

${ }^{17}$ La notion «d'oscillation pendulaire » s'applique au personnage seul en scène qui doit faire un choix à l'issue d'un monologue d'hésitation. Ce choix définit la relation qu'aura ce personnage à la Nouvelle Loi. Cf. Erich AuERBACH, Mimesis. La Représentation de la réalité dans la littérature occidentale, Paris, Gallimard, «Tel », 1992 (1 $1^{\text {ère }}$ éd. allemande 1946), p. 52-53.

${ }^{18}$ Le Procès de Paradis est l'épisode du Mystère de la Passion d'Arnoul Gréban au cours duquel les quatre filles de Dieu (allégories des vertus divines) s'affrontent dans une disputatio. À l'issue de cet épisode, l'Incarnation est décidée pour la Rédemption de l'Humanité. Arnoul Gréban écrit toute la scène du Procès de Paradis certainement en s'appuyant sur un des sermons de saint Bernard de Clairvaux, le sermon de l'Annonciation. Cf. SAINT Bernard De Clairvaux, Sermo 1 de Annuntiatione, in Sancti Bernardi opera, J. LeCleRCQ, H. Rochais, C.H. TALBOT (éd.), Rome, Ed. Cistercienses, t. V, 1968, p. 13-29. Toutes les références essentielles à cet épisode ont été réunies par Jean Pierre BORDIER, Le Jeu de la Passion. Le message chrétien et le théâtre français (XIII ${ }^{\mathrm{e}}-\mathrm{XVI}^{\mathrm{e}}$ siècles), Paris, Champion, «Bibliothèque du $\mathrm{XV}^{\mathrm{e}}$ siècle », 58,1998 , note 34 , p. 191. 
Ces doutes résonnent constamment avec le rapport de l'individu chrétien à la douleur et à la mort. La représentation appelle chaque spectateur à suivre l'exemple de Marie : si le doute est permis par la souffrance, l'acceptation dans la foi est la seule issue théologique possible, voire la seule issue dramatique pour le personnage de Marie. D'ailleurs, le planctus Mariae joue un véritable rôle dans la trame dramatique puisque, si seul l'Évangile de Jean mentionne la veille de Marie au pied de la croix ${ }^{19}$, tous les mystères de la Passion s'en souviennent et ne manquent pas de représenter cette scène. Au plan théâtral, le planctus Mariae revêt donc une importance capitale car tous les questionnements liés à la Passion du Fils ${ }^{20}$ sont doublés et soutenus par les déplacements de la Vierge sur le hourd, les vers interrogateurs devenant autant de didascalies internes dirigeant son jeu $^{21}$. Remarquons que les déplacements de Marie sont tellement révélateurs qu'ils convergent finalement vers la croix, soulignant l'acceptation de son rôle de mère souffrante.

Les doutes de Marie, qui amorcent «l'oscillation pendulaire » et qui sont le moteur du planctus Mariae, génèrent donc un jeu de scène et d'acteur : les déplacements de Marie soutiennent et traduisent les errements qui la divisent entre ses rôles d'Ancilla et de Mater Dolorosa. Cependant, ce personnage ne sort jamais de son profil biblique, mais acquiert seulement une plus grande latitude d'expression. Marie devient une sorte d'intercesseur entre le public et la Passion du Christ, le porte-parole potentiel du doute du spectateur sur la nécessité de la Passion et des souffrances intenables auxquelles il se doit d'assister s'il veut rester intégré au paradigme christique; le spectateur chrétien doit aussi recevoir la Passion comme une nécessité absolue.

\footnotetext{
${ }^{19}$ Cf. Jean, XI, 2.

${ }^{20}$ Cf. Le Mystère de la Passion d'Arnoul Gréban, éd. cit., v. 21989-21994.

${ }^{21}$ Ibid., v. 21390-21392.
} 


\section{Les doutes de Judas : une charnière}

Pour autant, d'autres modèles ou contre-modèles s'érigent devant le public du Mystère de la Passion de Jean Michel $^{22}$, notamment l'épisode des doutes de Judas, qui a été largement étudié par Stéphanie Le Briz-Orgeur ${ }^{23}$. En réalité, il s'agit de deux monologues n'appartenant pas au même espace discursif mais qu'on ne peut pas dissocier puisqu'ils peuvent se lire et surtout se comprendre de manière cursive $\mathrm{e}^{24}$. Le cheminement théologique et dramatique qui se construit au fil de ces monologues constitue une véritable charnière entre les doutes exprimés par le paradigme christique et ceux exprimés par le paradigme diabolique. Le premier extrait développe deux aspects majeurs. Un premier mouvement, au cours duquel Judas s'interroge sur lui-même au cœur du système christique, fait entendre une série de questions directes (mais rhétoriques) qui traduit l'expression du doute $^{25}$. Les réponses sont différées peu après ${ }^{26}$ et éloquentes : Judas reporte la faute sur le diable. Cette position est théologiquement intéressante car elle souligne la prédestination du personnage voué à la trahison. Au plan dramatique, cette posture est dangereuse. En effet, ce passage pourrait être «oublié »du public : quelle nécessité d'entendre les hésitations de celui qui est le traître depuis toujours? Par ailleurs, accepter inconditionnellement que Judas trahisse uniquement par soumission au diable le dédouane de toute responsabilité, et surtout, par là, on admettrait que l'apôtre ne s'interroge jamais sur la capacité de pardon du Christ.

\footnotetext{
22 JEAn Michel, Le Mystère de la Passion (Angers, 1486), Omer Jodogne (éd.), Gembloux, J. Duculot, 1959.

${ }^{23}$ Stéphanie Le BrIZ-ORGEUR, «Les monologues d'hésitation dans la Passion d'Arnoul Gréban », in Jean-Pierre BORDIER (dir.), Langues, codes et conventions dans l'ancien théâtre européen. Actes de la $3^{\text {ème }}$ rencontre sur l'ancien théâtre européen (1999), Paris, Champion, «Le Savoir de Mantice », 8, 2002, p. 149-166.

${ }^{24}$ JEAN MiCHEL, Le Mystère de la Passion, op. cit.. Les deux passages se trouvent respectivement v. 17474-17567 et v. 19065-19170.

${ }^{25}$ Ibid., v. 17518-17529.

${ }^{26}$ Ibid., v. 17536-17553.
} 
Davantage que l'attention du spectateur, c'est alors la visée d'édification théologique du mystère qui se perdrait (au plan du spectacle et du discours, donc de l'exemple de la mauvaise conversion qu'incarne Judas). En effet, ce monologue permet au fatiste de montrer le cheminement sur la voie de la damnation. De la narration (subjective) qui ouvre l'extrait à la décision de la trahison ultime, on assiste à la perdition du personnage. D'ailleurs, la proportion de vers exprimant le doute est bien inférieure à celle dédiée à la fatalité, à la colère et au désespoir : le discours des mauvais est à l'œuvre. Message théologique clair, ce passage amorce également la trame événementielle, puisqu'il s'achève sur une didascalie déclarant que Judas se rend auprès des Pharisiens pour l'ultime et fatale négociation.

Si les personnages d'Ève et de Marie doutaient, donc défiaient l'œuvre de Dieu, leurs doutes construisaient le choix de Dieu en conscience. Face à elles, Judas doute du pardon du Christ et son choix final est celui des diables. Le monologue de Judas apparaît ainsi comme un discours charnière entre le discours du doute du paradigme christique et celui du paradigme diabolique. D'ailleurs, le deuxième extrait de l'intervention de Judas poursuit son questionnement au point qu'on hésite à trancher : n'appartient-il déjà plus à la sphère christique ou est-il déjà membre de la mansion diabolique ${ }^{27}$ ? De la même manière que Gabriel intervient pour orienter les doutes de Joseph vers la certitude de Dieu, Désespérance et quelques diables entrent en jeu pour diriger Judas. Ces

\footnotetext{
${ }^{27}$ Nous utilisons ici le terme de «mansion » pour désigner les structures sur la scène qui représentent l'espace propre à un personnage ou à un groupe de personnages. Sur le sujet, voir Gustave COHEN, Études d'histoire du théâtre en France au Moyen Âge et à la Renaissance, Paris, Gallimard, 1956, chap. IV ; Graham A. RunNALLS, « Mansion and Lieu : Two Technicals Terms in Medieval French Staging ?», French Studies, 25 (1981), p. 385-393, repris dans Études sur les mystères, Paris, Champion, «Champion-varia », 14, 1998, p. 467-478 ; Michel Rousse, « Mystères et farces à la fin du Moyen Âge : problèmes de théâtre populaire », in La Scène et les tréteaux. Le théâtre de la farce au Moyen Âge, Orléans, Paradigme, «Medievalia », 50, 2004, p. 229-260.
} 
derniers le somment de ne plus douter mais Judas tourne ses questions vers Désespérance : «Las, que doy je faire ? / Me fault il deffaire ? $»^{28}$.

On pourrait penser que le dialogue qui s'engage avec Désespérance dédouane Judas de la décision qu'il prend. Nous pensons, au contraire, que Désespérance apparaît comme un écho de l'état d'esprit de Judas ${ }^{29}$. Cette démarche ouvre la possibilité d'une voix trinitaire au sein de la scène, et Judas s'exprimerait en fait suivant trois statuts : celui du mauvais (tombé en damnation), celui de l'apôtre (qui est en train de disparaître au fil des répliques) et celui du personnage de Judas ${ }^{30}$. L'étude de la deixis personnelle sert notre propos car chez Jean Michel, au cours des vers 19141-19150, les «deux Judas » se livrent à une joute verbale : «Que faistu ? Je suis obstiné $»^{31}$. C'est un dialogue interne qui se joue entre «l'apôtre » et « le mauvais » au cœur du personnage de Judas. La dernière réplique qui dit l'obstination de Judas fait basculer le propos et met fin au doute : seule la voix du personnage unifié parle pour dire sa trahison. Cette réunification acte la damnation finale. Dorénavant la prise de parole de Judas est émaillée des expressions diaboliques du langage comme peut le montrer la répétition de l'expression en despit ${ }^{32}$.

\footnotetext{
${ }^{28}$ JEAN MiCHEL, Le Mystère de la Passion (Angers, 1486), éd. cit., v. 21869-21870.

29 Désespérance traduit le caractère de Judas à ce moment-là, la préfixation est révélatrice: il n'a plus aucun espoir. Stéphanie Le Briz-Orgeur remarque que «le monologue de Judas, contrairement à ceux d'Ėve, de Joseph ou de Marie-Madeleine, se présente comme un "monologue éclaté" ». Selon elle, il s'agit davantage d'une «dispute in articulo mortis »; en d'autres termes, plus que s'adressant à lui-même seulement, Judas se divise en lui et aussi en Désespérance. Cf. Stéphanie LE BRIZORGEUR, «Les monologues d'hésitation dans la Passion d'Arnoul Gréban », art. cit., p. 162.

${ }^{30}$ Par cette expression neutre, nous voulons définir ce qu'est Judas tout au long du drame: un personnage qui oscille sans cesse, et jusqu'au bout, entre les deux paradigmes opposés sur le hourd. Même lorsqu'il est appelé à devenir un apôtre, son passé de meurtrier, souligné par les fatistes, le condamne déjà par prédestination.

${ }^{31}$ JEAN MiCHEL, Le Mystère de la Passion (Angers, 1486), éd. cit., v. 19150.

${ }^{32}$ Cette expression est utilisée par le diable pour établir la règle du jeu de dés, jeu de hasard diabolique pour lequel il faut à chaque lancer dire «en despit » d'une valeur divine. Ainsi, Satan engage Grifon à blasphémer à chaque coup de dé (ibid., v. 28125-28142).
} 
Par conséquent, les doutes de Judas sont une articulation évidente entre la certitude de la foi de l'apôtre et la certitude diabolique du damné. L'expression de ces doutes, si elle ne sert pas le propos dramatique (chacun connaît la destinée de Judas), construit le cheminement théologique et soutient l'identification théâtrale. En effet, Judas est le mauvais exemple à ne pas suivre, étant donné que ses doutes l'emmènent loin de Dieu. Ainsi, la confrontation de ces différents épisodes redessine la définition première du doute : finalement, le doute en lui-même n'est pas la défiance envers Dieu, il est le libre-arbitre en actes et en paroles et c'est le choix induit par les doutes du chrétien qui dit le choix de Dieu. Il apparaît donc logique - et, pourquoi pas, salvateur - de douter dans le mystère de la Passion.

\section{Le paradigme diabolique : entre doutes et certitudes}

Le paradigme diabolique, selon toute logique, devrait incarner cette notion de doute comme rejet de Dieu. À l'inverse, comme leur nature les détermine, les diables devraient aussi ne jamais douter et être les chantres de la certitude. En réalité, les diables adoptent les deux postures dans les mystères de la Passion, selon les directions voulues par le fatiste. D'abord, les doutes diaboliques ouvrent sur la discussion théologique. En effet, les diables doutent d'abord de la nature de Jésus. Arnoul Gréban place deux fois le mot doute dans la bouche de Satan pour exprimer le questionnement qui agite l'enfer à propos de la déité du Christ, traduisant par là le discours du mauvais ${ }^{33}$. Plus loin, Lucifer demande à sa cohorte : «Où est son corps ? $»^{34}$. C'est une question inattendue qui suppose qu'après avoir été abusée par l'Esprit de Jésus, la maisnie infernale cherche à appréhender son ennemi par les sens et, pourquoi pas, à le vaincre. L'indétermination des diables consiste aussi à discuter de la Rédemption lorsque le chant des

\footnotetext{
${ }^{33}$ Le Mystère de la Passion d'Arnoul Gréban, éd. cit., v. 10470-10474.

${ }^{34}$ Ibid., v. 28818.
} 
Justes résonne en enfer. Lucifer doute de la venue éventuelle du Christ pour libérer les Justes lors de la Descente en Enfer alors que celle-ci est pourtant annoncée par Satan ${ }^{35}$. Cette incertitude, vite balayée par Lucifer, souligne d'une part le ridicule diabolique et son ignorance de la décision du Procès de Paradis; d'autre part, elle insiste sur la méconnaissance générale de Lucifer, c'est-à-dire de celui qui est censé être le plus sage de la maisnie infernale. Cette ignorance est encore accentuée par le doute émis par Astaroth et Béric, pourtant témoins directs de l'Ascension : ils doutent de ce qu'ils ont $\mathrm{vu}^{36}$. Cela montre bien que les diables n'ont pas accès aux manifestations divines comme les personnages christiques: le message théologique est sauf. Finalement, porteurs du message chrétien tout autant que les autres personnages, les diables participent à l'élaboration de la visée d'édification du mystère de la Passion. Ils pensent comme des éléments intrinsèques au paradigme chrétien et émettent des doutes comme les autres personnages.

De plus, les doutes diaboliques apparaissent aussi comme une matrice dramatique. Par exemple, lorsqu'aux questions des démons sur l'identité de Marie répondent les hésitations de Satan, les doutes ne sont pas acceptés et enclenchent un violent «torchon $»^{37}$. Ou encore, lorsque Lucifer ne se satisfait pas des doutes sataniques, il requiert le départ de son messager pour la terre, ce qui appelle un déplacement scénique ${ }^{38}$. Ces échanges diaboliques autour des doutes construisent également la relation entre Lucifer et Satan et donnent de l'ampleur aux personnages ${ }^{39}$. Ainsi, les

\footnotetext{
${ }^{35}$ Ibid., v. 39918-39931.

${ }^{36}$ Ibid., v. 33210-33224.

${ }^{37}$ Ibid., v. 7323-7338. Le «torchon » est une scène diabolique au cours de laquelle la violence physique et verbale de plusieurs diables se déchaîne contre un autre diable de la maisnie infernale (ce diable étant très souvent Satan).

${ }^{38}$ Ibid., v. 39918-39931.

39 Rappelons que les scènes de diableries qui mettent Satan au cœur de l'action se structurent toujours de la même façon. D'abord, Satan l'ambassadeur infernal, de retour en enfer, doit faire un rapport à Lucifer, mais bien souvent ses renseignements sont de
} 
doutes de Satan génèrent de la peur en lui et cette peur le pousse à agir : il veut savoir pour s'apaiser ${ }^{40}$. De son côté, Lucifer refuse le doute satanique et prend les autres diables à témoin de l'incompétence de Satan, construisant alors l'unité de la maisnie infernale. Enfin, les doutes exprimés lors des conseils diaboliques agissent sur le déroulement ou l'arrêt de l'action scénique : l'attention du public se porte sur la mansion infernale. Là, les conséquences immédiates de l'expression des hésitations diaboliques sont que les diables ne s'enquièrent plus de nouvelles âmes et que les tortures cessent en enfer.

Cependant, la particularité diabolique est de s'inscrire en opposition à la sphère divine et au paradigme christique : les diables doivent donc aussi ériger la certitude face aux doutes des autres personnages. Ainsi, les doutes sataniques qui entourent la nature du Christ ne peuvent pas durer. Satan tente donc une définition alors qu'il ne sait rien (à la différence des autres personnages qui bénéficient de l'aide de Gabriel ou de Désespérance). La conclusion de Satan s'établit sur ce qui semble être sa raison, et il annonce, sûr de lui : «Jesus est quelque chose ${ }^{41}$. C'est l'antithèse absolue d'une définition. Ici Satan donne l'impression de réfléchir par lui-même : c'est l'expression d'un pseudo-libre-arbitre qui n'a pas lieu d'être théologiquement ${ }^{42}$ mais qui, au plan dramatique, construit le comique grotesque. La première appréhension de la certitude diabolique

piètre qualité, voire inexistants. Lucifer appelle alors les autres diables à constater l'incompétence de Satan et déclenche sur lui un "torchon ». S'ensuit un débat au sein de l'enfer sur la suite à donner à l'action diabolique puis, lorsque la décision est prise, Satan repart pour la terre, non sans requérir la «bénédiction » de Lucifer. Ces scènes sont presque toutes construites sur le même modèle et tendent à démontrer qu'il existe une relation particulière entre Lucifer et Satan, mais aussi que l'action diabolique est importante dans le mystère de la Passion; enfin, ces scènes confirment à la fois la violence qui règne en enfer et l'aspect grotesque des diables.

${ }^{40}$ Cf. Le Mystère de la Passion d'Arnoul Gréban, éd. cit., v. 7104-7106 et v. 10467-10468.

${ }^{41}$ Ibid., v. 10475.

42 Satan ne dispose pas du libre-arbitre contrairement aux hommes. Il a bien évidemment perdu cette faculté après la Chute des Anges. 
revient donc à comprendre que le diable n'accepte pas les doutes, traducteurs de son ignorance.

L’autre épisode révélateur de la certitude diabolique est celui de la préparation de la Descente aux Enfers. Cerbère non seulement ne doute jamais de la venue du Christ, mais encore est certain de le vaincre ${ }^{43}$. La surenchère belliqueuse de Cerbère refusant les quelques doutes de Satan et Lucifer ridiculise l'enfer encore davantage. En effet, à peine cette assurance est-elle exprimée que le Christ brise les portes de l'enfer : la débandade scénique est totale et la victoire de l'Esprit du Christ ${ }^{44}$ est rendue plus éclatante. C'est bien d'abord un rire lié à leur ridicule que déclenche la certitude des diables - mais pas uniquement. Le rire créé par des certitudes qui ne valent que pour les diables permet aux spectateurs de se libérer un peu de la crainte que ceux-ci pourraient susciter. D'abord, l'inexactitude des certitudes infernales laisse comprendre au spectateur qu'il possède une connaissance supérieure à celle des diables. Par exemple, lorsque les démons se demandent où est le Christ ${ }^{45}$, le spectateur chrétien possède la connaissance théologique et son expérience de la représentation pour connaître la réponse : Satan croit que le Christ est au tombeau tandis que le public attend la Descente aux Enfers. Le spectateur chrétien prend l'ascendant sur le diable qui ne fait plus aussi peur. Néanmoins, reconnaître la certitude diabolique c'est aussi se souvenir de la création des diables et du fait que la certitude de soi est considérée comme l'Orgueil. En effet, en relisant les certitudes de Lucifer juste avant la Chute des Anges, on comprend que cet ange est l'incarnation même de l'Orgueil ${ }^{46}$. D'ailleurs, le fatiste montre bien que la sanction divine vise essentiellement la

\footnotetext{
${ }^{43}$ Cf. Le Mystère de la Passion d'Arnoul Gréban, éd. cit., v. 26093-26095.

${ }^{44}$ Dans le Mystère de la Passion d'Arnoul Gréban, la didascalie précise que c'est bien «l'Esperit » de Jésus qui agit puisque son corps se trouve toujours physiquement sur la croix (Le Mystère de la Passion d'Arnoul Gréban, éd. cit., v. 26112).

${ }^{45}$ Ibid., v. 28805-28806.

${ }^{46}$ Ibid., v. 128-133.
} 
suppression de ce sentiment. Ainsi, tout de suite après la Chute des Anges, on assiste au monologue de lamentation luciférien. Cette tirade est émaillée de questions et de doutes sur son avenir ${ }^{47}$. La Chute des Anges, sanction divine, transforme les certitudes de l'ange en doutes du roi des enfers. Ces doutes resteront d'ailleurs des questions à l'adresse de Satan tout au long du mystère de la Passion.

Enfin, parfois, la certitude diabolique est nettement moins comique. Par exemple, lorsque Satan assure que ses plans pour perdre les hommes réussiront, il a bien souvent raison. En outre, il faut accepter que lorsque Satan confirme ses renseignements, il devient le premier des apôtres de la sphère divine ${ }^{48}$ et se trouve côte à côte avec le spectateur chrétien. En d'autres termes, par l'affirmation de ce qu'il a vu ou entendu (par exemple concernant les vertus de Marie), il prête foi aux vérités divines et devient une voix nouvelle pour asseoir la visée d'édification du mystère.

L'expression du doute dans les mystères de la Passion n'est pas anecdotique. De l'entourage christique aux diables, de nombreux personnages doutent. Si le premier mouvement est de tendre à considérer le doute comme une défiance envers Dieu, finalement les diverses occurrences de l'expression du doute redéfinissent cette notion. Les formes et les objectifs dramatiques de ces doutes sont différents selon qu'ils préoccupent les personnages du paradigme christique, Judas ou les diables, mais ils expriment toujours le libre-arbitre en actes et en paroles. C'est le choix qui met fin au doute et qui est porteur du message théologique et de la visée d'édification de l'œuvre; c'est ce choix qui caractérise l'appartenance du personnage à l'un ou l'autre camp sur le hourd. Judas est exemplaire de cette démarche : d'abord apôtre qui s'interroge à l'instar

\footnotetext{
${ }^{47}$ Ibid., v. 205-214.

${ }^{48}$ Ibid., v. 31785-31790.
} 
d'Ève ou de Marie, il finit par basculer, sous la force de la certitude, dans la sphère diabolique. Restent les diables, qui à la fois doutent et expriment la certitude. Complexes, parfois contradictoires mais toujours plus libres par rapport aux Écritures grâce à la plume du fatiste, les diables incarnent incontestablement la voie à ne pas suivre tant ils sont certains de vouloir être les ennemis de Dieu. Toutefois, porteurs du message chrétien comme les autres personnages, ils sont ceux dont les doutes sont autant de prétextes à la discussion théologique.

Les auteurs du théâtre religieux du Moyen Âge utilisent donc le doute comme une clé de compréhension de la structure qu'ils mettent en place ; ils permettent surtout au public de légitimer ses doutes éventuels tout en lui indiquant la seule issue salvatrice. À n'en pas douter, les fatistes ont offert une place de choix au spectateur chrétien : la place de celui qui ne doute jamais de l'issue du drame et qui peut s'assurer de sa possible Rédemption. 